\title{
Olfactory dysfunction in degenerative ataxias
}

\author{
T Connelly, J M Farmer, D R Lynch, R L Doty
}

J Neurol Neurosurg Psychiatry 2003;74:1435-1437

Several lines of evidence suggest that the cerebellum may play a role in higher-order olfactory processing. In this study, we administered the University of Pennsylvania Smell Identification Test (UPSIT), a standardised test of olfactory function, to patients with ataxias primarily due to cerebellar pathology (spinocerebellar ataxias and related disorders) and to patients with Friedreich ataxia, an ataxia associated mainly with loss of afferent cerebellar pathways. UPSIT scores were slightly lower in both patient groups than in the control subjects, but no differences were noted between the scores of the Friedreich and the other ataxia patients. Within the Friedreich ataxia group, the smell test scores did not correlate with the number of pathologic GAA repeats (a marker of genetic severity), disease duration, or categorical ambulatory ability. UPSIT scores did not correlate with disease duration, although they correlated marginally with ambulatory status in the patients with cerebellar pathology. This study suggests that olfactory dysfunction may be a subtle clinical component of degenerative ataxias, in concordance with the hypothesis that the cerebellum or its afferents plays some role in central olfactory processing.

O Ifactory dysfunction is present in a number of neurological disorders, including Alzheimer's disease (AD), schizophrenia, multiple sclerosis (MS), and idiopathic Parkinson's disease (PD). ${ }^{12}$ In PD, the prevalence of smell loss is greater than the prevalence of tremor, a cardinal motor sign of the disease. ${ }^{12}$ Olfactory testing is useful in differentiating $\mathrm{AD}$ from major affective disorder, ${ }^{4}$ and PD from such disorders as progressive supranuclear palsy and essential tremor. ${ }^{56}$ Such testing has been incorporated into a standardised diagnostic test battery for PD. ${ }^{7}$ With the exception of MS, the physiological basis for the olfactory loss of neurological disorders is poorly understood. In MS, olfactory dysfunction is strongly and inversely related to the number of active plaques within the primary and secondary olfactory cortices. ${ }^{89}$

To date, olfaction has not been tested in patients with degenerative ataxias. Interest in such ataxias stems not only from a desire to obtain a broader assessment of neurological diseases in which olfactory function is compromised, but from recent evidence that the cerebellum may play some role in olfactory function. ${ }^{10}$ The human cerebellum, which has traditionally been viewed as a motor coordinating centre, contains more neurones than the remainder of the brain, and is structurally connected with all major subdivisions of the central nervous system, including the cerebrum, basal ganglia, diencephalon, limbic system, brainstem, and spinal cord..$^{10}$ Support for the possibility that the cerebellum plays a role in olfactory processing stems from four sources: (a) early reports of olfactory deficits in patients with tumours in or near the cerebellum; ${ }^{12}{ }^{13}(b)$ indications of cerebellar anomalies in some disorders, such as schizophrenia, known to be associated with smell loss, ${ }^{14}$ (c) evidence that the staggered mutant mouse, a mouse with functional deficits in the olivocerebellar pathway, is hyposmic; ${ }^{15}$ and $(d)$ recent functional magnetic resonance imaging (fMRI) studies that show marked odour-induced cerebellar activity that is independent of sniffing. ${ }^{10}{ }^{16}$ In the fMRI work, odour-induced activation was found in the posterior lateral cerebellar hemispheres, with odour discrimination tasks activating superior semilunar lobule and posterior quadrangular lobule regions. Sniffing alone activated the right central lobule and the anterior vermis regions. Similar sensory:motor dissociations of activity between the anterior and posterior regions of the cerebellum have been noted in cognitive tasks involving auditory, auditory-spatial, visual, visuospatial, cutaneous, and tactile-spatial information processing. ${ }^{17}$

The purpose of the present study was to examine olfactory function in patients with diseases known to influence cerebellar activity to differing degrees, and to establish whether such function differs from that of matched normal controls. We also compared the function of patients with ataxias primarily due to cerebellar pathology (spinocerebellar ataxias and related disorders) to that of patients with Friedreich ataxia (FRDA), a disorder largely associated with degeneration of the afferent cerebellar pathways. ${ }^{18}$

\section{METHODS}

\section{Subjects}

We evaluated 35 patients with assorted degenerative ataxias. Diagnosis was confirmed by DNA testing for those patients with genetic diseases. Four further patients presented with idiopathic progressive ataxia and MRI-documented cerebellar degeneration with no apparent medical causes such as vitamin deficiencies, infections, or exposure to toxins, including alcohol. Two of these patients exhibited probable autosomal recessive inheritance, one autosomal dominant inheritance, and one sporadic disease. All patients were identified through the Neurology and Neurogenetics Clinic of the University of Pennsylvania Medical Center. Of the patients studied, only one patient (who had spinocerebellar ataxia 3 (SCA3)) reported active use of alcohol (two drinks per day) and only one other patient (who also had SCA3) was diabetic. While nasal endoscopy was not performed, no patients were noted to have a history of nasal difficulties, sinusitis, or structural abnormalities of the nasopharynx on historical review or cranial imaging studies. Clinical data included the disease duration at testing, as well as the ambulatory status of the individual. Ambulatory status was categorised as unassisted, assisted, or wheelchair bound. The length of the shorter pathological GAA repeat in the FRDA gene was also tabulated for FRDA patients. The normal

Abbreviations: $A D$, Alzheimer's disease; $F M R I$, functional magnetic resonance imaging; FRDA, Friedreich ataxia; MS, multiple sclerosis; PD, Parkinson's disease; SCA, spinocerebellar ataxia 
controls were randomly selected from a large database maintained at the University of Pennsylvania Smell and Taste Center, and were individually matched to the ataxia patients on the basis of age, gender, and current smoking habits.

The patients were separated into two groups for analysis. ${ }^{19}$ One group consisted of the 23 FRDA patients. This group contained 11 men and 12 women (mean (SD) age: 20.46 (12.78) and 35.92 (13.15) years, respectively). A second group (designated CNS ataxias) was comprised of patients with other ataxias consistent with cerebellar degeneration, including autosomal dominant spinocerebellar atrophies. This group contained two women (aged 36 and 46 years) with SCA2; one man (aged 54 years) and four women (mean (SD) age: 50.40 (7.77) years) with SCA3 (Machado-Joseph Disease); one man with SCA7 (aged 62 years); and four women (mean (SD) age 44.25 (16.07) years) with unidentified forms of cerebral degeneration. None of the SCA3 patients had progressed to the point where obvious cognitive dysfunction was present, exhibiting normal scores on the Picture Identification Test (PIT) (all scores > 38). The PIT is a 40 -item test specifically designed to screen individuals unable to perform the non-olfactory components of the University of Pennsylvania Smell Identification Test (UPSIT), ${ }^{20}$ the olfactory test employed in this study. ${ }^{21}$

Four patients of the cerebellar degeneration group exhibited significant dystonic features on examination, and four individuals of the cerebellar degeneration group had a history of depression at some point during their illness.

\section{Olfactory test procedure}

The UPSIT was administered bilaterally to all patients, with the assistance of a trained technician. ${ }^{20}$ The administering technician noted no difficulties with sniffing in any patient. The UPSIT is a 40-odorant forced choice standardised microencapsulated test (commercially termed the Smell Identification Test $^{\text {TM }}$; Sensonics, Inc., Haddon Heights, NJ, USA) that has been shown to be highly reliable (test-retest $\mathrm{r}=0.94$ ) and sensitive to a wide range of olfactory deficits, including those associated with ageing, ${ }^{20}{ }^{22}$ smoking, ${ }^{23}$ and a number of neurodegenerative disorders. ${ }^{12}$ Before being enrolled into the program, subjects provided informed written consent, in accordance with the requirements of the University Committee on Studies Involving Human Beings.

\section{RESULTS}

\section{Friedreich ataxia $v$ controls}

The test scores of the FRDA group were significantly lower than those of their matched controls (fig lA). The magnitude of this effect, however, was modest (respective medians (interquartile range) $=36$ (4) and 38 (2); $Z=-5.921$, $\mathrm{P}<0.001$; Wilcoxon matched-pair signed-ranks test (WSRT)). A total of 17 of the 23 FRDA patients (70\%) scored below their matched controls, and five patients $(22 \%)$ fell into the range of moderate microsmia. ${ }^{24}$ Performance on the UPSIT did not correlate with the length of the shorter pathological expanded GAA triplet repeat in the FRDA gene (Spearman $\mathrm{r}=0.0 \mathrm{l}$; NS), disease duration $(\mathrm{r}=-0.0 \mathrm{l}$; NS), or ambulatory ability (unassisted, assisted, or wheelchairbound) $(\mathrm{r}=0.02$; NS)

\section{CNS ataxias $v$ controls}

UPSIT scores for the combined CNS ataxia group were also significantly lower than for their matched controls [respective medians (interquartile ranges) $=34.5$ (5.5) and 37 (3)], $[\mathrm{Z}=-4.297, \mathrm{P}<0.001$; WSRT; fig 1B). Again the magnitude

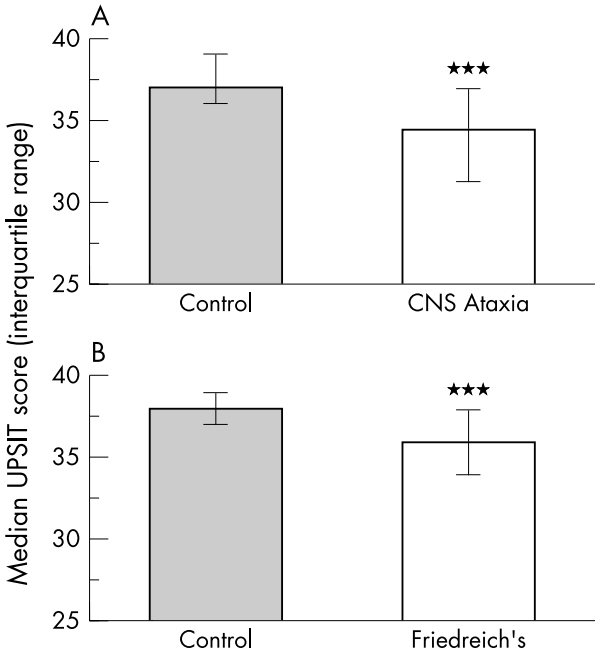

Figure 1 Median (IQR) number of correct items on the 40-item University of Pennsylvania Smell Identification Test (UPSIT) in patients with Friedreich ataxia $(A)$ and combined CNS ataxias (B), along with respective matched controls. ${ }^{* * *} p<0.001$ for Wilcoxon matched-pair signed-ranks test.

of this effect was small. Five of the twelve CNS ataxia patients $(42 \%)$ had UPSIT scores indicative of some degree of microsmia, and ten of the twelve $(83 \%)$ scored below their matched controls. UPSIT scores did not correlate with disease duration $(\mathrm{r}=0.05)$ and correlated only marginally with categorical ambulation status $(\mathrm{r}=-0.49, \mathrm{p}<0.11)$. While no meaningful differences in olfactory function appeared to be present between those patients with CNS ataxias based on depression or dystonia, the small sample sizes precluded formal statistical assessment.

\section{Friedreich ataxia $v$ cns ataxias}

A statistical comparison of UPSIT scores from the FRDA and CNS ataxia groups revealed no significant differences [respective medians (interquartile ranges) $=36$ (4) and 34.5 (7); Mann-Whitney $U=170, p=0.261]$. As 10 of the 12 subjects of the CNS group were women, compared with 12 of 23 in the FRDA group, we also examined whether the UPSIT scores differed only for the women of these two groups. Again, no significant difference emerged [respective medians (interquartile range) $=38$ (1) and 35.5 (6); $U=84$, $\mathrm{p}=0.104]$. Although the ages of the two groups were not comparable, they fell within the range where age has little or no influence on UPSIT scores. ${ }^{24}$

\section{DISCUSSION}

Overall, this study suggests that patients with Friedreich's ataxia or degenerative CNS ataxias perform, on average, more poorly than age- and gender-matched normal controls on a standardised test of odour identification. The observed decrements, however, are quite small and far less marked that those found in $\mathrm{AD}$, idiopathic $\mathrm{PD}$, schizophrenia, and some other neurodegenerative diseases, where UPSIT scores typically fall below 25. None of the patients were anosmic, and only two reported being conscious of any smell loss; the majority had UPSIT scores falling within the normal range noted on the test. ${ }^{24}$ The abnormalities generally did not correlate well with disease duration or categorical ambulatory status, suggesting that these statistically significant decrements reflect a true sensory difference rather than problems 
in taking the test related to motor abilities. Nevertheless, the possibility cannot be entirely ruled out that both sensory and motor factors could be responsible for the subtle differences that are observed.

Although the UPSIT scores of patients with FRDA were slightly higher than those of individuals with CNS ataxias, these differences were not statistically significant. However, the pattern of abnormalities is consistent with the underlying pathology of these disorders. While the major pathological abnormality of FRDA is located in afferent proprioceptive senses, afferent visual, auditory and small fiber somatosensory functions are also affected in FRDA. These deficits do not always correlate with the duration or degree of ataxia in the disorder. ${ }^{25}$ Thus, a subtle loss of olfactory sensations without correlation with ambulatory function is consistent with the global loss of sensory pathways in FRDA. In contrast, the individuals in the group we designated as CNS ataxias share the unifying feature of cerebellar degeneration, most likely from different pathophysiologies and without uniform primary sensory deficits. Hence, their olfactory dysfunction more likely reflects primary cerebellar damage and thus correlates better with the loss of ambulation.

\section{ACKNOWLEDGEMENTS}

Supported by NIH Research Grants PO1 DC 00161, RO1 DC 04278, RO1 DC 02974, RO1 AG 17496 (RLD) and a Beeson Scholar Award (DRL) from AFAR.

\section{Authors' affiliations}

T Connelly, J M Farmer, D R Lynch, R L Doty, Smell and Taste Center, Department of Otorhinolaryngology: Head and Neck Surgery, University of Pennsylvania School of Medicine, Philadelphia, PA 19104, USA

Correspondence to: Dr R L Doty, Smell and Taste Center, 5 Ravdin Pavilion, University of Pennsylvania Medical Center, 3400 Spruce Street, Philadelphia, PA 19104, USA; e-mail: doty@mail.med.upenn.edu

\section{REFERENCES}

1 Doty RL. Odor perception in neurodegenerative diseases and schizophrenia. In: Doty RL, ed. Handbook of olfaction and gustation, 2nd ed. New York: Marcel Dekker, 2003:479-502.

2 Mesholam RI, Moberg PJ, Mahr RN, et al. Olfaction in neurodegenerative disease: a meta-analysis of olfactory functioning in Alzheimer's and Parkinson's diseases. Arch Neurol 1998;55:84-90.
3 Doty RL, Deems D, Stellar S. Olfactory dysfunction in Parkinson's disease: A general deficit unrelated to neurologic signs, disease stage, or disease duration. Neurology 1988;38:1237-44.

4 McCaffrey RJ, Duff K, Solomon GS. Olfactory dysfunction discriminates probable Alzheimer's dementia from major depression: a cross-validation and extension. J Neuropsychiat Clin Neurosci 2000;12:29-33.

5 Doty RL, Golbe LI, McKeown DA, et al. Olfactory testing differentiates between progressive supranuclear palsy and idiopathic Parkinson's disease. Neurology 1993;43:962-5.

6 Busenbark KL, Huber SI, Greer G, et al. Olfactory function in essential tremor. Neurology 1992;42:1631-2.

7 Montgomery EB Jr, Koller WC, LaMantia TJ, et al. Early detection of probable idiopathic Parkinson's disease: I. Development of a diagnostic test battery. 2000;15:467-473.

8 Doty RL, Li C, Mannon L, et al. Olfactory dysfunction in multiple sclerosis. New Engl J Med 1997;336:1918-19.

9 Doty RL, Li C, Mannon U, et al. Olfactory dysfunction in multiple sclerosis: Relation to longitudinal changes in plaque numbers in central olfactory structures. Neurology 1999;53:880-2.

10 Sobel N, Prabhakaran V, Hartley CA, et al. Odorant-induced and sniffinduced activation in the cerebellum of the human. $J$ Neurosci 1998; 18:8990-9001.

11 William R, Herrup K. The control of neuron number. Ann Rev Neurosci 1988;11:423-53.

12 Tucker BR. Report of a case of tumor of the ponto-cerebellar angle on the left side of the brain with bilateral loss of smell and disturbance of taste. Old Dominion J Med Surg 1911;XIII:327-34.

13 Peregud, GM. Anosmia in tumors of the fourth ventricle. Russk Oto-laringol 1931;24:101-5.

14 Tran KD, Smutzer GS, Doty RL, et al. Reduced Purkinje cell size in the cerebellar vermis of elderly patients with schizophrenia. Am J Psychiatry 1998; 155:1288-90.

15 Deiss V, Baudoin C. Hyposmia for butanol and vanillin in mutant staggerer mice. Physiol Behav 1997;61:209-13.

16 Yousem D, Williams SCR, Howard RO, et al. Functional MR imaging during odor stimulation: preliminary data. Radiology 1997;204:833-8.

17 Parsons LM, Fox PT. Sensory and cognitive functions. Int Rev Neurobiol 1997;41:255-271.

18 Pandolfo M. Molecular pathogenesis of Friedreich's ataxia. Arch Neurol 1999;56(10):1201-8.

19 Scarano V, de Cristofaro T, De Michele G, et al. Serum transferring receptor levels in Friedreich's and other degenerative ataxias. Neurology 2001;57:159-160.

20 Doty RL, Shaman P, Dann M. Development of the University of Pennsylvania Smell Identification Test: A standardized microencapsulated test of olfactory function. Physiol Behav (Monograph) 1984;32:489-502.

21 Vollmecke T, Doty RL. Development of the Picture Identification Test (PIT): A research companion to the University of Pennsylvania Smell Identification Test. Chem Senses 1985;10:413-14.

22 Doty RL, Shaman P, Applebaum SL, et al. Smell identification ability: Changes with age. Science 1984;226:1441-3.

23 Frye RE, Schwartz B, Doty RL. Dose-related effects of cigarette smoking on olfactory function. JAMA 1990;263:1233-6.

24 Doty RL. The Smell Identification Test ${ }^{T M}$ administration manual, 3rd ed. Haddon Heights, NJ: Sensonics, 1995.

25 Wenzel W, Camacho L, Claus D, et al. Visually evoked potentials in Friedreich's ataxia. Adv Neurol 1982;32:131-9. 